\title{
ARTICLE
}

\section{Trace amine-associated receptor 1 agonism promotes wakefulness without impairment of cognition in Cynomolgus macaques}

Anushka V. Goonawardena ${ }^{1}$, Stephen R. Morairty ${ }^{1}$, Ryan Dell ${ }^{1}$, Gabriel A. Orellana ${ }^{1}$, Marius C. Hoener iD $^{2}$, Tanya L. Wallace ${ }^{1,3}$ and Thomas S. Kilduff (iD ${ }^{1}$

Trace amine-associated receptor 1 (TAAR1) is a G-protein coupled receptor with affinity for the trace amines. TAAR1 agonists have pro-cognitive, antidepressant-, and antipsychotic-like properties in both rodents and non-human primates (NHPs). TAAR1 agonism also increases wakefulness and suppresses rapid-eye movement (REM) sleep in mice and rats and reduces cataplexy in two mouse models of narcolepsy. We investigated the effects of TAAR1 agonism in Cynomolgus macaques, a diurnal species that exhibits consolidated night-time sleep, and evaluated the effects of TAAR1 agonists on cognition using a working memory (WM) paradigm in this species. Adult male Cynomolgus macaques $(n=6)$ were surgically implanted to record the electroencephalogram (EEG), electromyogram, and locomotor activity (LMA) and the efficacy of the TAAR1 partial agonist RO5263397 $(0.1,1,10 \mathrm{mg} / \mathrm{kg}, \mathrm{p} .0$.$) on$ sleep/wake, EEG spectra, and LMA was determined. In a second experiment, the acute effects of RO5263397 (0.1,1,10 mg/kg, p.o.) were assessed on a delayed-match-to-sample test of WM in adult male macaques $(n=7)$. RO5263397 $(10 \mathrm{mg} / \mathrm{kg}) \mathrm{administered} \mathrm{at}$ lights off, when sleep pressure was high, promoted wakefulness and reduced both REM and non-REM sleep without inducing hyperlocomotion. RO5263397 (10 mg/kg) also increased delta/theta activity during all vigilance states. RO5263397 had no effect on WM at either short $(2 \mathrm{sec})$ or long $(10 \mathrm{sec})$ delay intervals. The wake-enhancing and REM-suppressing effects of R05263397 shown here in a diurnal primate are consistent with previous results in nocturnal rodents. These effects and the associated alterations in EEG spectra occurred without inducing hyperlocomotion or affecting WM, encouraging further study of TAAR1 agonists as potential narcolepsy therapeutics.

Neuropsychopharmacology (2019) 44:1485-1493; https://doi.org/10.1038/s41386-019-0386-8

\section{INTRODUCTION}

Trace amines (TAs), such as $\beta$-phenylethylamine (PEA), $p$-tyramine, octopamine, and tryptamine, represent a group of endogenous amines closely related to the classical biogenic amines [1-3]. TAs, synthesized in neuronal terminals from the same amino acid precursors as dopamine (DA), noradrenaline (NE), and serotonin (5-HT) by aromatic L-amino acid decarboxylase (AADC), are colocalized and co-released with biogenic amine transmitters and rapidly metabolized by monoamine oxidase (MAO) A and B [3]. TAs are endogenous ligands for trace amine-associated receptor 1 (TAAR1), a G-protein coupled receptor (GPCR) that negatively modulates monoaminergic and glutamatergic activity [4-6]. TAAR1 is expressed in cortical, midbrain, and limbic forebrain regions important for behavioral arousal and motivation $[5,7,8]$. Selective TAAR1 full and partial agonists have been shown to exhibit pro-cognitive, antidepressant, antipsychotic-like in rodents and non-human primates (NHPs) [9, 10]. In addition, TAAR1 partial agonists also have been shown to increase wakefulness and suppress Rapid Eye Movement (REM) sleep in both rats $[9,10]$ and mice [11] dose-dependently and to reduce cataplexy in two mouse models of narcolepsy [12].
Although TAAR1 agonists increase vigilance in rodents, these animals are nocturnal and have polyphasic sleep/wake cycles in comparison to the consolidated periods of sleep and wakefulness characteristic of both humans and NHPs. Among NHPs, sleep/ wake patterns have previously been studied in squirrel monkeys $[13,14]$, rhesus macaques [15-18], juvenile, and adult Cynomolgus macaques [15-21] and other species [22]. The similar sleep/wake architecture of NHPs and humans has resulted in testing of preclinical compounds in NHPs for efficacy as well as safety prior to initiation of clinical studies $[18,23]$. In a recent study, we demonstrated that the adenosine receptor antagonist caffeine enhanced wakefulness nearly four-fold during the dark phase with consequent reductions in both non-rapid eye movement (NREM) and REM sleep, decreased slow wave activity (SWA) during wakefulness, and increased higher electroencephalographic (EEG) frequency activity during NREM sleep in adult Cynomolgus macaques [21].

The prefrontal cortex is critical for cognitive processes such as executive function and working memory (WM), and both processes rely heavily on monoaminergic arousal systems. The functional relationship between TAAR1 and the monoamines

\footnotetext{
${ }^{1}$ Center for Neuroscience, Biosciences Division, SRI International, Menlo Park, CA 94025, USA and ${ }^{2}$ Neuroscience, Ophthalmology and Rare Disease DTA, pRED, Roche Innovation Center Basel, F. Hoffmann-La Roche Ltd, Basel, Switzerland

Correspondence: Thomas S. Kilduff (thomas.kilduff@sri.com)

${ }^{3}$ Present address: Blackthorn Therapeutics, 780 Brannan St, San Francisco, CA 94103, USA
}

Received: 26 January 2019 Revised: 27 March 2019 Accepted: 27 March 2019

Published online: 6 April 2019 


\section{6}

$[7,8,24]$ led us to previously investigate the effects of TAAR1 agonists on executive function (e.g., response inhibition, planning), and our results showed an improvement in this domain in both NHPs and in rodents [9, 10]. Given the involvement TAAR1 in regulating dopamine release $[7,8]$, it was of interest to investigate additional PFC-mediated cognitive domains sensitive to monoaminergic tone that can be affected under conditions of disease, aging, and stress.

Since TAAR1 agonists have been shown to alter sleep/wake activity and EEG spectral composition in rodents, here we assessed the acute effects of the TAAR1 partial agonist, RO5263397, on nocturnal sleep/wake activity in middle-aged, adult (9-11 years) Cynomolgus macaques, a diurnal species that exhibit consolidated night-time sleep. We also extended our evaluation of RO5263397, tested at the same doses, to investigate WM, a cognitive domain that has not been assessed previously in this species with a TAAR1 agonist.

\section{MATERIALS AND METHODS}

All experimental procedures were approved by the Institutional Animal Care and Use Committee at SRI International and in compliance with National Institutes of Health guidelines for the care and use of laboratory animals. Details are described in Supplemental Information.

Animals

Adult male cynomolgus macaques (Macaca fascicularis; 7-10 kg) were maintained in constant environmental conditions (temperature $21 \pm 3^{\circ} \mathrm{C}$; humidity $30-70 \% ; 12: 12 \mathrm{~h}$ light:dark cycle with lights on at 06:00 and off at 18:00). All animals received a full daily regimen of standard certified commercial chow (Purina Animal Nutrition, Gray Summit, MO) supplemented with fresh fruits and vegetables and had access to water ad libitum in their home cage. Animals were individually housed but had visual, auditory and olfactory contact with other monkeys throughout the study. Videos and music were played in the housing room to provide environmental enrichment during the day.

\section{Surgical procedures}

Animals were fully anesthetized [telazole $(4 \mathrm{mg} / \mathrm{kg}, \mathrm{i} . \mathrm{m}$.$) and 1 \%$ isoflurane] and a disk-shaped telemetry transmitter (D70-EEE; DSI, St-Paul, MN) was placed between the internal oblique muscle and the aponeurosis of the transverse abdominis muscle as described previously [21]. EEG wires from the transmitter were placed in the left and right frontal cortices according to the 10-20 system in humans and referenced to electrodes placed over the occipital cortex [Fp1 (left)/Fp2 (right): AP $+18.0 \mathrm{~mm}, \mathrm{ML} \pm 15.0 \mathrm{~mm}$; Oz reference: AP $-22.0 \mathrm{~mm}, \mathrm{ML} \pm 3.0 \mathrm{~mm}$ (left/right) from bregma]. Stainless steel screws were inserted until the tips were estimated to touch the dura mater and the exposed EEG wire tips were wrapped around the four screws; silver epoxy was then applied over the wire/screws to ensure good electrical conductivity. Once the epoxy was fully dried ( 15-20 min), all wires/screws were insulated with dental cement (FuliCEM resin modified glass ionomer cement; Net 32 Inc., Cary, NC). Electromyographic (EMG) electrodes connected to the transmitter were implanted bilaterally into the superficial neck musculature (trapezius). All animals received perioperative anesthetic monitoring, postoperative analgesia for $\geq 72 \mathrm{~h}$, antibiotics for 10-14 days, and were allowed a minimum of 21 days recovery period before experiments.

Electrophysiological and locomotor activity recording

Telemetry receiver boards (RPC-1; DSI, St. Paul, MN) were mounted individually to each home cage to facilitate real-time EEG/EMG recording. Continuous acquisition of the EEG and EMG telemetry signals occurred at a sampling rate of $500 \mathrm{~Hz}$ using DataQuest A.R.
T. (version 4.36; DSI); the frequency range of the D70-EEE telemetry transmitters was $0.3-100 \mathrm{~Hz}$. Locomotor activity (LMA) (counts $/ 15 \mathrm{sec}$ ) was collected through Actical accelerometers (Philips Respironics, NV) that were attached to each animal's collar. All data are presented according to Zeitgeber Time (ZT) in which $\mathrm{ZTO}=$ lights on and ZT12 = lights off.

\section{Experimental design and dosing procedures}

Animals were acclimated to the dosing procedure for multiple sessions with $0.3 \%$ Tween 80 in sterile water (po) prior to start of each experiment. Two experiments were conducted:

Experiment 1: acute effects of TAAR1 partial agonist, RO5263397 on sleep/wake during the initial $6 \mathrm{~h}$ of the dark phase. In this experiment, the acute effects of RO5263397 (0.1, 1, and $10 \mathrm{mg} / \mathrm{kg}$, p.o.; dose volume: $2.5 \mathrm{ml} / \mathrm{kg}$ ) and vehicle $(0.3 \%$ Tween 80 in sterile water) administered 30 min prior to lights-off on EEG, EMG, and LMA were assessed in six macaques during the initial $6 \mathrm{~h}$ of the dark phase. The doses of R05263397 (synthesized at Roche) were chosen based on a previous study [10]. Since animals were housed and recorded in the same room, the delivery of each dose was not randomized: to avoid the potential sleep disruption due to different treatment conditions (e.g., interference of sleep in vehicle-treated animals by wakefulness induced in R05263397-treated animals), all animals received the same dose on any given night. A $7 \mathrm{~d}$ washout period elapsed between treatments.

Experiment 2: effects of TAAR1 partial agonist RO5263397 on a DMTS working memory task. In this experiment, the effects of RO5263397 (0, 0.1, 1, $10 \mathrm{mg} / \mathrm{kg}$, p.o.) on Delayed-match-tosample (DMTS) performance were assessed in a separate cohort of seven adult male macaques 30 min after administration at ZT3. As in Experiment 1, a 7-day washout period was allowed between treatments.

\section{Vigilance state analysis}

Vigilance states were determined by visual inspection of EEG and EMG signals using Profusion (Compumedics USA, Charlotte, NC) according to the American Academy of Sleep Medicine (AASM) guidelines for human sleep scoring $[25,26]$ and adapted for cynomolgus macaques as described previously [21]. Trained investigators classified each $30 \mathrm{sec}$ epoch as one of five states: WAKE, NREM sleep stages N1, N2, or N3, or REM sleep. WAKE was scored when $>50 \%$ of the epoch consisted of alpha $(8-13 \mathrm{~Hz})$ activity or low amplitude, mixed frequency $(2-7 \mathrm{~Hz})$ activity, and active EMG. N1 (Stage 1) was scored when $50 \%$ of the epoch consisted of relatively low amplitude, mixed frequency $(2-7 \mathrm{~Hz})$ activity and $<50 \%$ of the epoch contained alpha $(8-13 \mathrm{~Hz})$ activity accompanied with lower EMG activity. N2 (Stage 2) was scored when K-complexes (1-2 Hz isolated waves) and/or sleep spindles (regular $12-16 \mathrm{~Hz}$ EEG sequences) were observed and $<20 \%$ of the epoch contained high amplitude $(>75 \mu \mathrm{V})$, low frequency $(1-4 \mathrm{~Hz})$ activity. N3 (Stages $3+4$ ) was scored when $\geq 20 \%$ of the epoch consisted of high amplitude $(>75 \mu \mathrm{V})$, low frequency $(1-4 \mathrm{~Hz})$ EEG waves (i.e., slow wave activity). REM was scored when the epoch contained relatively low voltage, mixed frequency activity with predominant theta activity $(4-9 \mathrm{~Hz})$ accompanied with low EMG activity (i.e., atonia). Epochs with prominent muscular artifacts were identified and excluded from subsequent EEG spectral analysis as described below.

\section{EEG spectral analysis}

Spectral analysis of EEG data was computed offline using a Fast Fourier transform algorithm (MATLAB, Natick, MA) on all epochs without artifacts. Fourier analysis was limited to frequencies $<100 \mathrm{~Hz}$ due to the frequency bandwidth of the D70-EEE transmitters. Contiguous frequencies were binned into standard 
frequency bands (delta: $0.3-4 \mathrm{~Hz}$; theta: $4-8 \mathrm{~Hz}$; alpha: $8-12 \mathrm{~Hz}$; sigma: $12-16 \mathrm{~Hz}$; beta: $16-30 \mathrm{~Hz}$; low gamma: $30-50 \mathrm{~Hz}$ and high gamma: $50-100 \mathrm{~Hz}$ ). Artifact removal proceeded as described previously [21]. For Experiment 1 , the $F_{25}, F_{50}$, and $F_{75}$ values were calculated for each animal (where $F=$ total EEG power of a 6 -sec sub-epoch and $F_{25}, F_{50}$, and $F_{75}$ refer to the 25 th, 50th, and 75th percentiles of the total distribution of EEG power values) during the initial dark phase following vehicle treatment and then applied to all $6 \mathrm{sec}$ sub-epochs for both vehicle and RO5263397 conditions.

Spectral data for each $30 \mathrm{sec}$ epoch was comprised of the average of all artifact-free $6 \mathrm{sec}$ sub-epochs. Thereafter, the artifact-free absolute power following each of the RO5263397 treatments was normalized to each animal's average power following vehicle measured during the initial 6-h of the dark phase. Subsequently, the mean normalized power for each frequency band (delta, theta, alpha, sigma, beta, low gamma and high gamma) within each vigilance state (WAKE, NREM and REM) was calculated for each animal before group analyses occurred. Overall, $9.4 \pm 1.2,9.5 \pm 1.1,7.7 \pm 1.5$, and $11.3 \pm 2.9 \%$ of the epochs were removed as artifact from the recordings of the vehicle, 0.1, 1, and $10 \mathrm{mg} / \mathrm{kg}$ RO5263397-treated animals, respectively.

Delayed-match-to-sample paradigm

Macaques $(n=7)$ were seated in standard restraint chairs and placed into sound-attenuated chambers equipped with a monitor delivering visual stimuli (Cambridge Neuropsychological Test Automated Battery (CANTAB] ver. 9.4; Lafayette Instruments, Lafayette, IN) as described previously [27]. For this test, monkeys were presented with a single sample stimulus consisting of a complex pixelated pattern comprised of four colors in the center of the screen that they must touch to begin the trial. In order to receive a food reward (190 mg banana pellet, Newco Distributors, Hayward, CA), the monkey had to correctly select the sample stimulus in the choice phase following a 2 or $10 \mathrm{sec}$ delay while a distractor stimulus was simultaneously presented. The distractor stimulus consisted of a stimulus comprised of the same colors but organized in a slightly different pattern. Each test session included 100 trials with a 2-sec inter-trial interval. The outcome measures were correct choices, correct choice reaction times, and omissions. While all correct responses were rewarded with a food pellet, incorrect responses and omissions were followed by a $10-\mathrm{sec}$ time-out period, during which the houselights were turned off and the animal was unable to earn a pellet. The use of two delay times provided a dynamic range to determine whether improvements and/or impairments in DMTS performance occurred following administration of RO5263397.

\section{Data analyses}

For Experiment 1, the duration of each vigilance state was calculated across the initial $6 \mathrm{~h}$ of the dark phase, both cumulatively and on an hourly basis (percent time). Sleep efficiency (\%), sleep onset latency (SOL), REM onset latency (ROL), wake after sleep onset (WASO), the number of awakenings, REM/NREM sleep ratios, and the number of NREM/REM cycles were calculated as described previously [21] and in Supplementary Information. Hourly LMA averages (counts/15 sec) were assessed for each treatment and the mean actograms were calculated by summing all the counts per $15 \mathrm{sec}$ sample and dividing by the number of $15 \mathrm{sec}$ samples within the $6 \mathrm{~h}$ period.

For Experiment 2, once animals achieved the performance criteria (i.e., accuracy of $\geq 75 \%$ at both delays for three consecutive sessions), the mean accuracy (i.e., correct choice trials/total number of trials $\times 100$ ), mean 'choice' reaction time (i.e., reaction time for all correct choice trials) and mean number of omissions (i.e., all trials the animal failed to respond to sample stimuli) were assessed following all treatments.
Statistical analysis

Data were analyzed using SigmaPlot (Systat Software Inc., San Jose, CA). All animals received all treatment conditions in Experiments 1 and 2. For Experiment 1, statistical significance was assessed on the group means for all sleep/wake parameters and LMA during the initial $6 \mathrm{~h}$ of the dark phase (18:30-00:30) following all treatments using one-way repeated measures (RM) ANOVAs followed by individual paired $t$-tests of treatment vs. the vehicle condition. The hourly percent time spent in WAKE, NREM, and REM was compared using two-way RM ANOVAs (factor 1: treatment; factor 2: time) followed by pairwise multiple comparison (Fisher) tests. Similarly, one-way RM ANOVAs followed by individual paired $t$-tests between vehicle and all RO5263397 treatments were conducted independently for each frequency band for normalized power spectra.

For Experiment 2, overall accuracy (\% correct), choice reaction time and the number of omissions were assessed with two-way

\section{A}

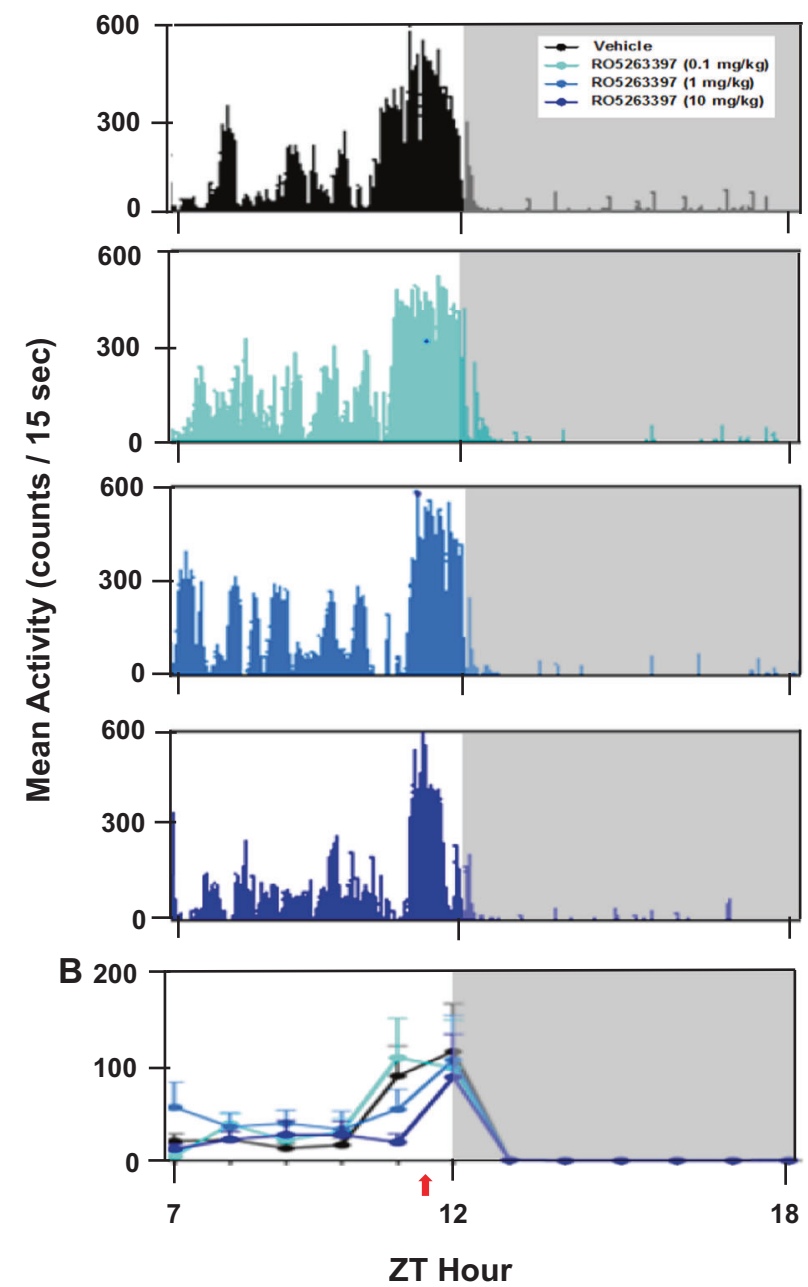

Fig. 1 Mean locomotor activity (LMA) recorded across a 12-h portion of the light and dark phases (ZT7-ZT18) for Cynomolgus macaques $(n=6)$ treated with either vehicle or the trace amineassociated receptor 1 (TAAR1) partial agonist, RO5263397. a Mean LMA (counts/15-sec) plotted as histograms following treatment with vehicle and three doses of RO5263397 $(0.1,1$, and $10 \mathrm{mg} / \mathrm{kg}$, p.o.). b Summary data for vehicle treatment and the three doses studied. Data are represented as mean \pm SEM. Red arrow below the abscissa indicates the time of dosing. A 7-day washout period elapsed between treatments. The higher LMA between ZT11 and ZT12 reflects the chairing of the macaques and movement to and from the dosing room by the experimenter $30 \mathrm{~min}$ prior to lights off 
Table 1. Sleep/wake characteristics of Cynomolgus macaques during the first $6 \mathrm{~h}$ of the dark phase after administration of vehicle or RO5263397 (0.1, 1 , and $10 \mathrm{mg} / \mathrm{kg}$, p.o.) $30 \mathrm{~min}$ before light offset in Experiment $1(n=6)$

\begin{tabular}{lcccc}
\hline Sleep-wake parameters & Vehicle (Mean \pm SEM) & RO5263397 (0.1 mg/kg) & RO5263397 (1 mg/kg) & RO5263397 (10 mg/kg) \\
\hline Time in darkness (min) & 360 & 360 & 360 & 360 \\
Total sleep time (TST; min) & $254.2 \pm 14.1$ & $251.8 \pm 20.0$ & $263.1 \pm 22.4$ & $164.3 \pm 14.7^{\mathrm{a}}$ \\
Sleep efficiency (\%) & $70.6 \pm 3.9$ & $69.9 \pm 5.6$ & $73.1 \pm 6.2$ & $45.6 \pm 4.1^{\mathrm{a}}$ \\
Sleep onset latency (SOL; min) & $22.0 \pm 3.1$ & $19.4 \pm 4.1$ & $17.8 \pm 4.5$ & $24.0 \pm 4.4$ \\
REM onset latency (ROL; min) & $93.5 \pm 12.7$ & $80.7 \pm 19.3$ & $87.3 \pm 21.1$ & $159.9 \pm 32.2$ \\
Wake after sleep onset (WASO; min) & $83.8 \pm 11.1$ & $88.8 \pm 18.1$ & $79.1 \pm 20.7$ & $171.7 \pm 13.4^{\mathrm{a}}$ \\
REM sleep duration (min) & $35.0 \pm 8.6$ & $38.8 \pm 4.6$ & $30.5 \pm 7.0$ & $4.8 \pm 2.7^{\mathrm{a}}$ \\
Stage N1 NREM duration (min) & $4.6 \pm 1.3$ & $6.2 \pm 1.6$ & $3.6 \pm 1.2$ & $2.0 \pm 1.3$ \\
Stage N2 NREM duration (min) & $152.1 \pm 13.5$ & $138.1 \pm 12.6$ & $156.0 \pm 18.7$ & $116.7 \pm 16.0$ \\
Stage N3 NREM duration (min) & $62.5 \pm 17.0$ & $68.7 \pm 16.1$ & $23.0 \pm 22.6$ & $40.8 \pm 4.4^{\mathrm{a}}$ \\
Cumulative NREM sleep duration (min) & $219.2 \pm 14.0$ & $212.9 \pm 19.9$ & $0.136 \pm 0.032$ & $159.5 \pm 12.4^{\mathrm{a}}$ \\
REM / NREM ratio & $0.166 \pm 0.047$ & $0.194 \pm 0.036$ & $49.5 \pm 9.4$ & $0.026 \pm 0.014^{\mathrm{a}}$ \\
Awakenings (\#) & $45.5 \pm 5.9$ & $50.2 \pm 5.2$ & $6.2 \pm 1.1$ & $58.5 \pm 6.8$ \\
NREM / REM Cycles (\#) & $6.3 \pm 1.1$ & $6.8 \pm 1.2$ & & $1.3 \pm 0.6^{\mathrm{a}}$ \\
\hline a $p \leq 0.05$ vs. vehicle & & & \\
\hline
\end{tabular}

RM ANOVAs (factor 1: treatment; factor 2: delay) followed by pairwise multiple comparison (Fisher) tests. All data are represented as mean \pm SEM. In all cases, significance was defined as $p \leq 0.05$; normality tests were conducted for all experiments.

\section{RESULTS}

Experiment 1: acute effects of TAAR1 partial agonist RO5263397 on sleep/wake during the initial $6 \mathrm{~h}$ of the dark phase

The average LMA collected from macaques $(n=6)$ recorded in their home cages from ZT7 to ZT18 indicated a clear diurnal pattern with the highest activity levels during the light phase; activity was essentially non-existent during the dark phase following vehicle and all RO5263397 treatments (Fig. 1a). For LMA, a main effect of time $\left(F_{5,75}=2.659, p<0.05\right)$ was evident without an effect of treatment or a treatment $\times$ time interaction.

Although LMA in their home cages was unchanged during the $6 \mathrm{~h}$ dark phase subsequent to treatment with R05263397 (Fig. 1b), highly significant effects on sleep parameters were observed after the $10 \mathrm{mg} / \mathrm{kg}$ treatment compared to vehicle (Table 1). Following vehicle, macaques showed a SOL of $22.0 \pm 3.1 \mathrm{~min}$ after light offset and an overall sleep efficiency of $70.6 \pm 3.9 \%$ during the initial $6 \mathrm{~h}$ of the dark phase. In contrast, R05263397 showed overall main effects on a number of sleep/ wake parameters including TST $\left(F_{(3,15}=13.12, p<0.001\right)$, WASO; $\left.F_{(3,15}=12.44, p<0.001\right)$, NREM duration $\left(F_{(3,15}=8.68, p<0.001\right)$, N3 duration $\left(F_{(3,15}=4.84, p<0.05\right)$, REM duration $\left(F_{(3,15}=7.95\right.$, $p<0.01)$; REM-NREM ratio $\left(F_{(3.15}=7.35, p<0.01\right)$ and the number of NREM-REM cycles $\left(F_{(3,15}=8.88, p<0.001\right)$. Post hoc analysis revealed that macaques treated with the $10 \mathrm{mg} / \mathrm{kg}$ dose of R05263397 showed large reductions in TST $(t=6.31, p<$ $0.001)$, sleep efficiency $(t=6.31, p<0.001)$, and more than twice the time WASO; $t=5.82, p<0.01)$ compared to vehicle treatment (Table 1) even though SOL was unchanged. Macaques treated with $10 \mathrm{mg} / \mathrm{kg}$ of RO5263397 also had significantly reduced NREM sleep $(t=4.52, p<0.01)$, particularly N3 sleep, compared to vehicle $(t=2.58, p<0.05)$, and REM sleep was almost completely suppressed $(t=3.00, p<0.05)$. As a result, the $10 \mathrm{mg} / \mathrm{kg}$-treatment resulted in fewer NREM-REM cycles $(t=$ $3.54, p<0.05)$ and a greatly reduced REM-NREM ratio $(t=2.57$, $p<0.05)$ compared to vehicle (Table 1 ). In a separate experiment with a narrower dose range $(1,3$, and $10 \mathrm{mg} / \mathrm{kg}$, p.o.) tested in a smaller number of subjects $(n=4)$, RO5263397 at $3 \mathrm{mg} / \mathrm{kg}$ significantly reduced N3 sleep $(t=10.84, p<0.01)$, and $10 \mathrm{mg} / \mathrm{kg}$ reduced both N3 sleep $(t=4.06, p<0.05)$ and the number of NREM-REM cycles $(t=3.18, p<0.05)$ (Supplementary Table 1$)$.

Figure 2 presents the individual hypnograms and the hourly distributions of WAKE, NREM, and REM sleep during the initial 6 $h$ of the dark phase for both vehicle- and RO5263397-treatment. The hypnograms (Fig. 2a, b) illustrate the RO5263397-induced wake increase and suppression of REM sleep following the $10 \mathrm{mg} / \mathrm{kg}$ dose for the majority of subjects. Overall, main effects of treatment $\left(F_{3,75}=13.119, p<0.001\right)$ and time $\left(F_{5,75}=8.217\right.$, $p<0.001)$ but no treatment $\times$ time interaction $\left(F_{15,75}=1.703\right.$, $p=0.068$ ) were evident during wakefulness (Fig. 2c). The overall increase in WAKE following the $10 \mathrm{mg} / \mathrm{kg}$ dose of RO5263397 was due to an increase from ZT15 to ZT18. Similarly, main effects of treatment $\left(F_{3,75}=8.684, p<0.001\right)$ and time $\left(F_{5,75}=4.891\right.$, $p<0.001)$ but no treatment $\times$ time interaction $\left(F_{15,75}=1.313\right.$, $p=0.216$ ) were observed for NREM sleep (Fig. 2d). Main effects of treatment $\left(F_{3,75}=7.950, p<0.01\right)$, time $\left(F_{5,75}=8.902\right.$, $p<0.001)$ and a treatment $\times$ time interaction $\left(F_{15,75}=1.863, p<\right.$ 0.05 ) were evident for REM sleep (Fig. 2e). Post hoc analysis revealed that the $10 \mathrm{mg} / \mathrm{kg}$ condition significantly decreased both NREM and REM sleep from ZT15 to ZT18 $(p<0.05)$. These results demonstrate that the high dose of RO5263397 increased wakefulness and reduced NREM and REM sleep; similar results were obtained in the study in which RO5263397 was tested at 1, 3 , and $10 \mathrm{mg} / \mathrm{kg}$ (Supplementary Fig. 1).

Spectral power within the conventional frequency bands was calculated from the frontal cortex for each vigilance state during the initial $6 \mathrm{~h}$ of the dark phase for the vehicle- and RO5263397treatments (Fig. 3). Figure 3a shows the absolute power spectral profile from $0.3-100 \mathrm{~Hz}$ for WAKE, NREM, and REM sleep. Figure $3 \mathrm{~b}$ presents the normalized power spectral profiles $(0.3-100 \mathrm{~Hz})$ for each vigilance state and dose of RO5263397 in which the absolute power values were normalized to vehicle for the $6 \mathrm{~h}$ period. RO5263397 at $10 \mathrm{mg} / \mathrm{kg}$ showed significant increases in delta and theta power compared to vehicle during all three vigilance states [WAKE (delta: $t=17.15 ; p<0.001$; theta: $t=18.02 ; p<0.001$ ); NREM (delta: $t=4.04 ; p<0.05$; theta: $t=7.27 ; p<0.01$ ), and REM sleep (delta: $t=4.19 ; \quad p<0.05$; theta: $t=4.28 ; p<0.05$ )]. In addition, RO5263397 increased delta power during NREM sleep 
A

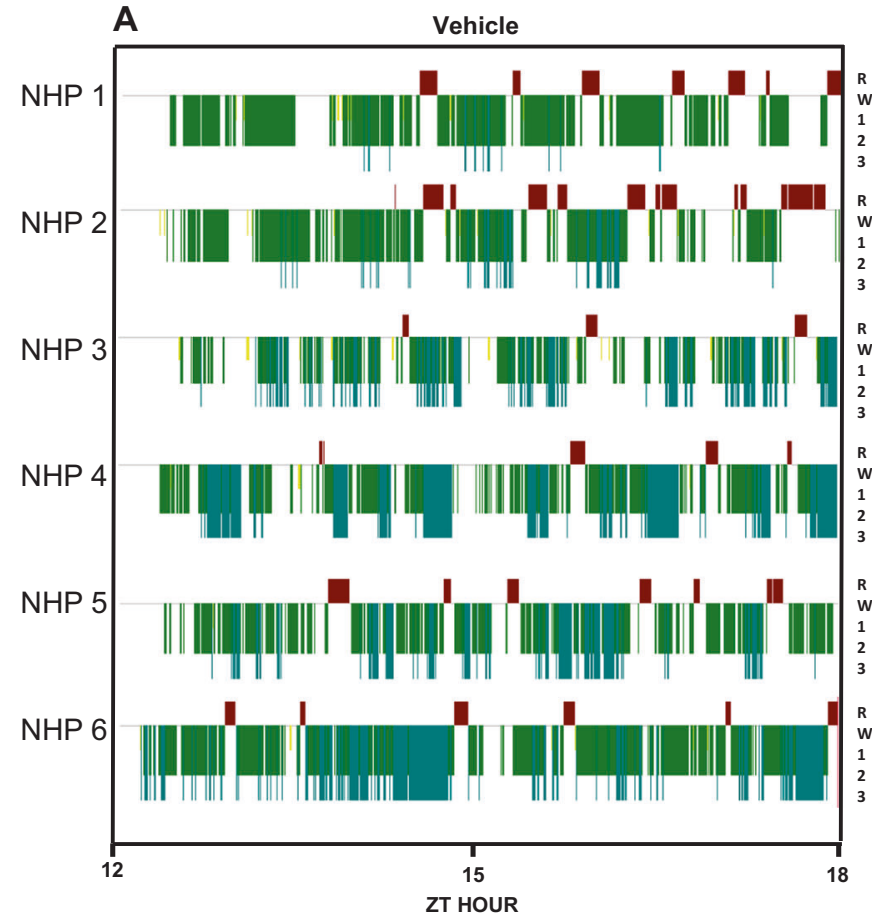

B

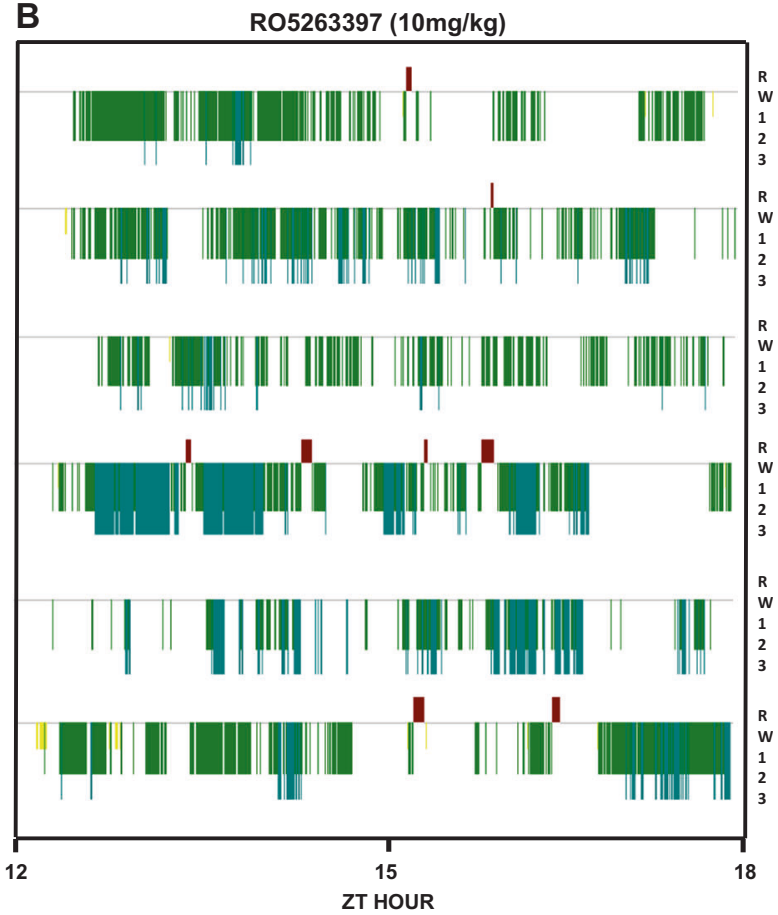

E

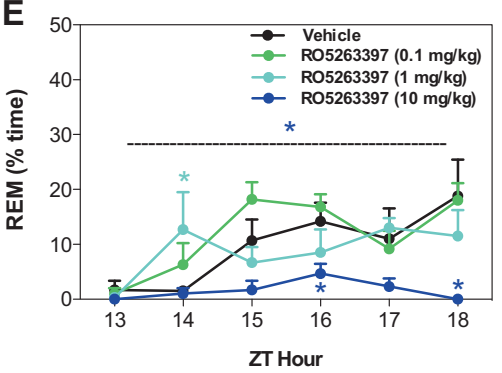

Fig. 2 Individual hypnograms from six Cynomolgus macaques during the initial $6 \mathrm{~h}$ dark phase (ZT13 to ZT18) following administration of a vehicle or $\mathbf{b}$ the trace amine-associated receptor 1 (TAAR1) partial agonist, RO5263397 (10 mg/kg, p.o.), 30 min before light offset (ZT11.5). Hourly distribution (\% time) of c Wakefulness d NREM, and e REM sleep during the same period following vehicle or RO5263397 (0.1, 1, and 10 $\mathrm{mg} / \mathrm{kg}$ ). RO5263397 at $10 \mathrm{mg} / \mathrm{kg}$ increased wakefulness while suppressing NREM and REM sleep. Data are mean \pm SEM $(n=6)$. ${ }^{*} p \leq 0.05$ (The color of asterisk denotes a significant difference between the respective dose vs. vehicle). A 7-day washout period elapsed between treatments. $\mathrm{R}=\mathrm{REM}$ (red); W=WAKE (flat line); $1=$ stage N1 (yellow); $2=$ stage N2 (green); $3=$ stage N3 (blue)

following both $0.1 \mathrm{mg} / \mathrm{kg}(t=4.98 ; p<0.01)$ and $1 \mathrm{mg} / \mathrm{kg}(t=3.76$; $p<0.05)$ and an increase in theta $(t=3.67 ; p<0.05)$ occurred during REM sleep following the $0.1 \mathrm{mg} / \mathrm{kg}$ dose compared to vehicle. Subtle decreases in low $(t=-2.81 ; p<0.05)$ and high gamma $(t=-4.08 ; p<0.05)$ during wakefulness were also evident following the $0.1 \mathrm{mg} / \mathrm{kg}$ dose (Fig. 3c). These results indicate that RO5263397 affects slow wave oscillations in the frontal cortex across all vigilance states during the initial $6 \mathrm{~h}$ dark phase in macaques.

Experiment 2: effects of TAAR1 partial agonist R05263397 on a DMTS working memory task

To determine whether R05263397 affects WM, macaques $(n=7)$ were trained to perform a DMTS task with two delay intervals ( 2 and $10 \mathrm{sec}$ ). Two-way RM ANOVA revealed the absence of main effects of treatment $\left(F_{3,18}=0.624, p=0.609\right)$, delay $\left(F_{1,18}=\right.$ $1.518, p=0.264)$ or a treatment $\times$ delay interaction $\left(F_{3,18}=0.834\right.$, $p=0.493$ ) on choice accuracy (Fig. 4a). Similarly, neither a main effect of treatment $\left(F_{3,18}=1.364, p=0.285\right)$ nor a treatment $x$ delay interaction $\left(F_{3,18}=0.118, p=0.948\right)$ was observed for 'choice' reaction time, although a delay effect was evident
$\left(F_{1,18}=16.910, p<0.01\right.$; Fig. $\left.4 b\right)$. As expected, post hoc analysis revealed that animals took longer to respond correctly when the delay was longer (10-sec) compared to shorter (2 sec) across all treatment groups. In addition, there was no effect of R05263397 treatment $\left(F_{3,18}=0.971, p=0.428\right)$, delay $\left(F_{1,18}=\right.$ $2.627, p=0.156)$ or a treatment $\mathrm{x}$ delay interaction $\left(F_{3,18}=0.688\right.$, $p=0.571$ ) on the number of omissions (Fig. 4c). A similar effect was observed when delay intervals were extended up to $100 \mathrm{sec}$ (Supplementary Information). These results indicate that the doses of RO5263397 used in Experiment 1 had little effect on DMTS performance.

\section{DISCUSSION}

Having previously shown that TAAR1 partial agonism has wakepromoting, pro-cognitive, antipsychotic-, and antidepressantlike activities, we have postulated that TAAR1 may represent a novel therapeutic target for pathological sleepiness and the treatment of neuropsychiatric disorders [9-12]. Moreover, having established the basal sleep/wake characteristics in Cynomolgus macaques [21], we sought to determine whether 

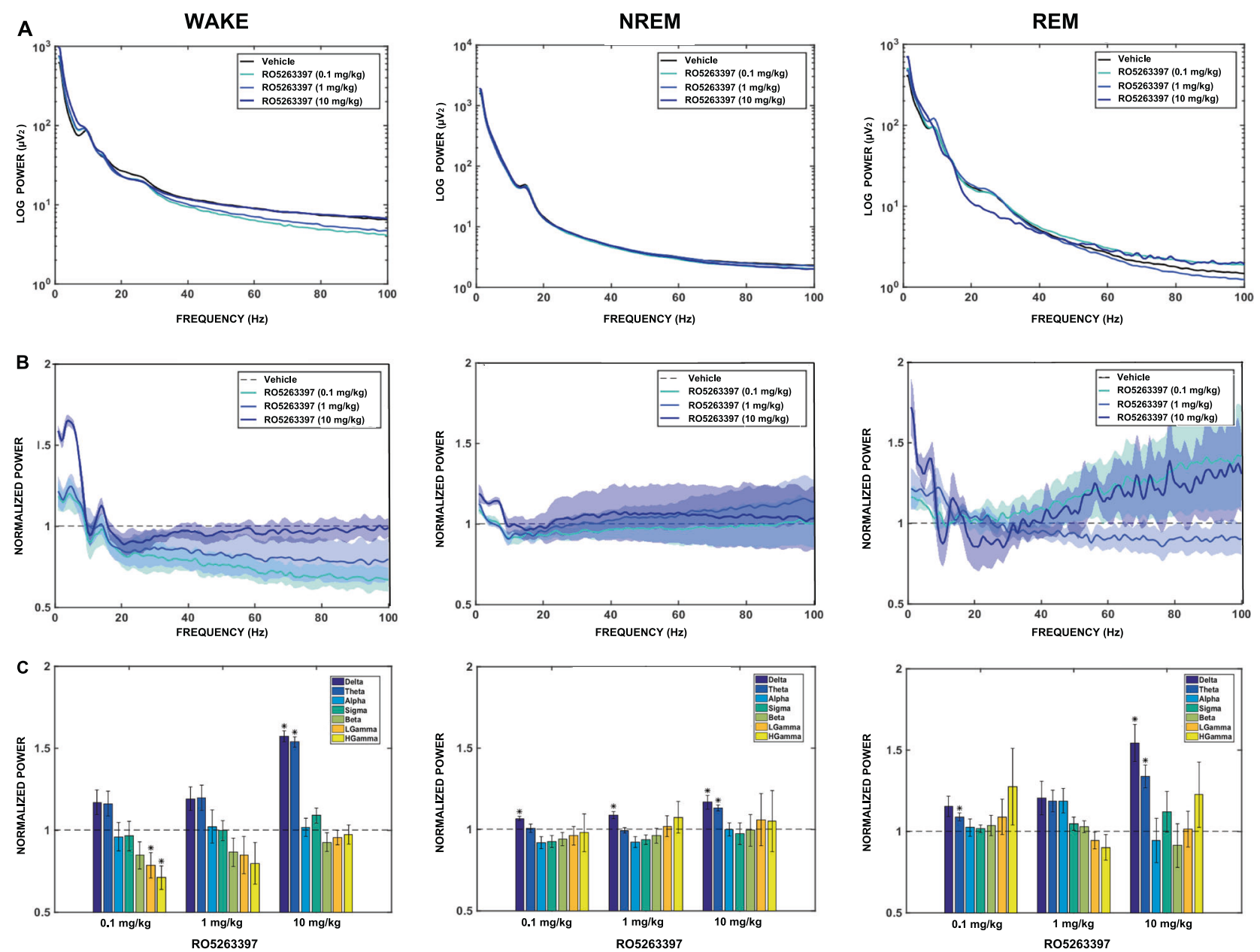

Fig. 3 The effects of the trace amine-associated receptor 1 (TAAR1) partial agonist RO5263397 on EEG spectra recorded during the initial 6-h of the dark phase in Cynomolgus macaques. a Absolute (Log) power spectrum from $0.3-100 \mathrm{~Hz}$ for WAKE, NREM and REM sleep during the initial $6 \mathrm{~h}$ of the dark phase following vehicle $(0.3 \%$ Tween 80 in sterile water) or RO5263397 (0.1, 1, 10 mg/kg, p.o.) treatment. b Normalized power spectrum from $0.3-100 \mathrm{~Hz}$ and c corresponding delta, theta, alpha, sigma, beta, low gamma, and high gamma (left to right) frequency bands for WAKE, NREM and REM sleep following RO5263397 treatment during same $6 \mathrm{~h}$ of the dark phase. RO5263397 at $10 \mathrm{mg} / \mathrm{kg}$ increased activity in the delta and theta bandwidths across all vigilance states (WAKE, NREM, and REM); delta activity during NREM sleep increased after all three doses. Data are mean \pm SEM $(n=5) .{ }^{*} p \leq 0.05$ (RO5263397 as compared to the vehicle condition). A 7-day washout period elapsed between treatments

the novel TAAR1 partial agonist RO5263397 affected vigilance states in this species when administered at a time of day when the homeostatic drive to sleep is high. Given the pro-cognitive effect of RO5263397 in primates tested on an object recognition task [10], we also sought to determine whether this compound affected WM in a DMTS test.

TAAR1 partial agonism and LMA

Macaques exhibited a clear diurnal pattern of activity with nearly continuous wakefulness during the light phase and virtually no LMA during the dark phase. Similar to rodents [11, 12], RO5263397-induced wakefulness was not accompanied by hyperactivity. The activity monitors, tagged to each animal's collar, are more sensitive to gross vs. fine locomotor movements. During the dark phase, macaques tended to sit upright on their perch and showed little movement while awake as opposed to during the light phase.

TAAR1 partial agonism promotes wakefulness

During the dark phase, the TAAR1 agonist RO5263397 $(10 \mathrm{mg} / \mathrm{kg})$ caused a two-fold increase in WASO, decreased NREM sleep (particularly N3 in which the highest SWA levels occur), and reduced REM sleep. As a result, macaques exhibited fewer NREM-REM cycles and a reduced REM-NREM ratio. The increased wakefulness was particularly evident around ZT15 of the dark phase, consistent with the $1.5 \mathrm{~h} T_{\max }$ following oral administration of this compound in Cynomolgus macaques [10]. These results are consistent with previous rodent studies that showed similar results at 0.3 and $1 \mathrm{mg} / \mathrm{kg}$ in mice $[11,12]$ and at 1 and 3 $\mathrm{mg} / \mathrm{kg}$ in rats [10]. Furthermore, such effects were completely absent in TAAR1 knockout (KO) mice, indicating TAAR1 dependence $[11,12]$, and were greatly exacerbated in TAAR1 overexpressing (OE) mice [11], suggesting that TAAR1 partial agonism increases waking drive.

TAAR1 partial agonists may increase wakefulness and promote arousal by increasing monoaminergic activity $[9,10]$ and modulating DA and 5-HT signaling in the ascending reticular activating system. TAAR1 has been shown to regulate monoamine transporters and to interact with DA D2 and 5-HT1A receptors [8, 28-30]. R05263397 dose-dependently increases firing rates of ventral tegmental area (VTA) dopaminergic and dorsal raphe nucleus (DRN) serotonergic neurons, mimicking the effects of the TAAR1 antagonist EPPTB $[30,31]$, thereby suggesting that this partial agonist may have antagonistic activity that blocks 
Vehicle

R05263397 (0.1 mg/kg)

R05263397 (1 mg/kg)

R05263397 (10 mg/kg)
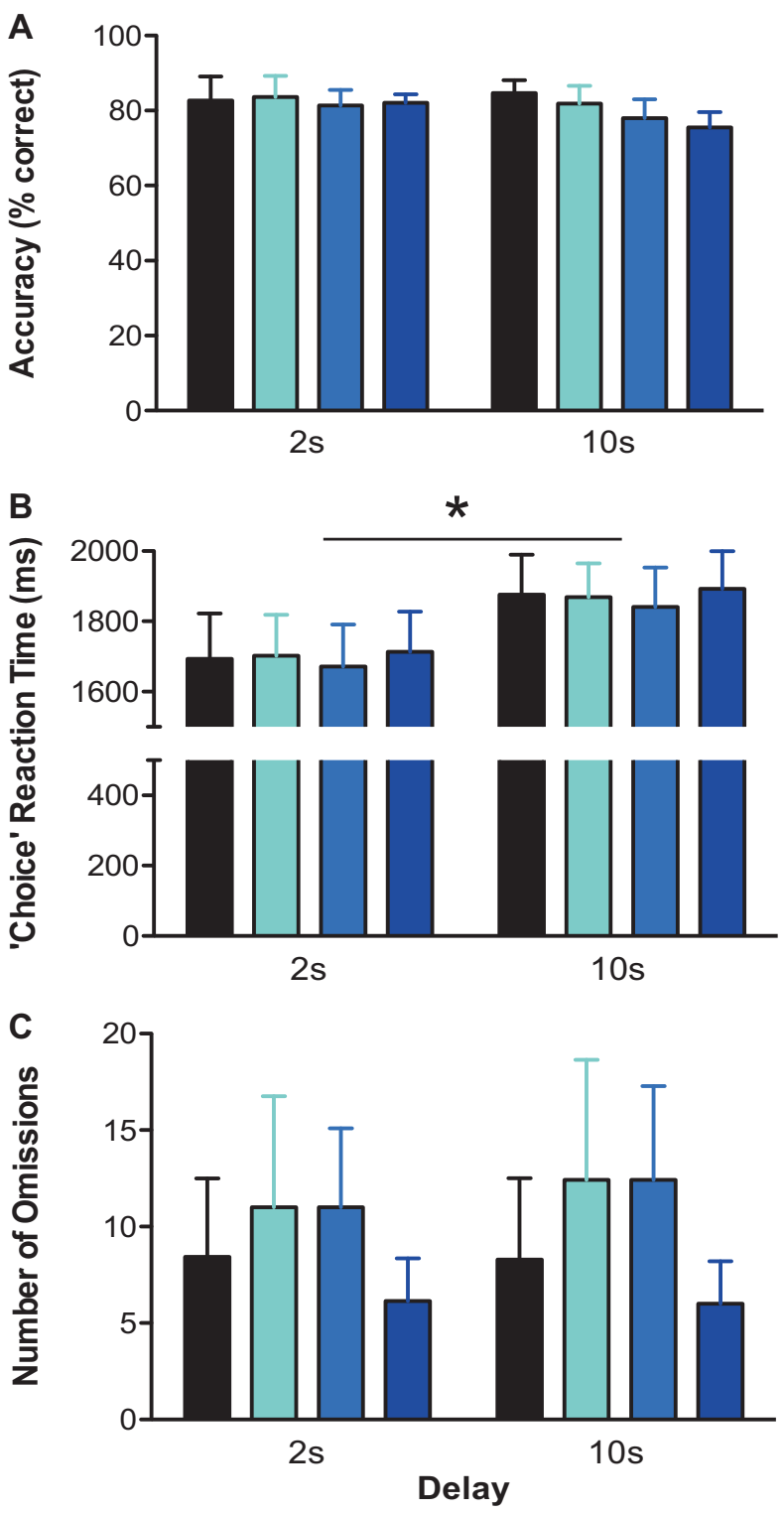

Fig. 4 Effects of the trace amine-associated receptor 1 (TAAR1) partial agonist RO5263397 on performance of the delayed-matchto-sample (DMTS) working memory task in Cynomolgus macaques. Overall a 'choice' accuracy (\% correct), b 'choice' reaction time, and c number of omissions during both 2 and $10 \mathrm{~s}$ delay intervals following the administration of vehicle $(0.3 \%$ Tween 80 in sterile water) or RO5263397 (0.1, 1, $10 \mathrm{mg} / \mathrm{kg}$, p.o.) $30 \mathrm{~min}$ prior to testing. No main effect of treatment or treatment $\times$ delay interaction were observed on any of the measures. A significant main effect of delay was observed on choice reaction time $\left({ }^{*} p \leq 0.05\right)$. Data are represented as mean \pm SEM $(n=7)$. A 7-day washout period elapsed between treatments

constitutive TAAR1 tone. Given that both DA and 5-HT are implicated in sleep/wake regulation, future studies should assess the relative contribution of each of these systems to TAAR1 agonist-induced wakefulness.
TAAR1 partial agonism and EEG spectra

The dose of RO5263397 (10 mg/kg) that enhanced wakefulness also increased delta and theta power in the frontal cortex in all three vigilance states during the initial $6 \mathrm{~h}$ dark phase. Furthermore, increased delta activity was evident during NREM sleep following 0.1 and $1 \mathrm{mg} / \mathrm{kg}$ and increased delta/theta activity occurred during REM following $0.1 \mathrm{mg} / \mathrm{kg}$. These results indicate that RO5263397 profoundly affects cortical activity by increasing the power of slow oscillations in the frontal cortex despite an increase in wakefulness in these macaques. These results contrast with those which we previously observed in mice in which acute decreases in alpha, beta and gamma activity occurred during waking and NREM sleep in the first $3 \mathrm{~h}$ following wake-promoting doses of 0.3 and $1 \mathrm{mg} / \mathrm{kg}$ [11]. Although we observed trends toward subtle reductions in alpha, sigma, beta, low and high gamma activity following 0.1 and $1 \mathrm{mg} / \mathrm{kg}$ doses during waking and NREM sleep in macaques, the only significant reductions were in high gamma following $0.1 \mathrm{mg} / \mathrm{kg}$ during waking. One possibility for this difference could be the fact that RO5263397-induced alterations in EEG spectra were assessed for the entire $6 \mathrm{~h}$ period of the dark phase here compared to the initial $3 \mathrm{~h}$ in the mouse study, and most spectral effects were eliminated by $4-6 \mathrm{~h}$ post-treatment in mice [11].

Since TAAR1 negatively modulates monoaminergic and glutamatergic activity $[4,5]$, one mechanism by which TAAR1 activation alters the observed EEG spectral activity may be through modulation of DA release [7]. The wake-promoting compound modafinil, known to block the dopamine transporter [32], caused a three-fold increase in theta $(7-11 \mathrm{~Hz})$ activity during waking in mice [33]. We have recently shown that modafinil increases delta power during both waking and NREM sleep in mice [34]. However, other non-DA mechanisms of action for modafinil have been suggested [35]. TAAR1 also modulates cortical NMDA receptormediated glutamate transmission [36]. Since NMDA receptor antagonists increase gamma [27, 37-40] and theta [39] power, TAAR1 modulation of glutamatergic neurotransmission may be a critical component underlying EEG activity.

TAAR1 partial agonism and WM

WM is a cognitive domain sensitive to disruption under conditions in which monoaminergic tone is increased non-optimally, e.g., either pharmacologically or by stress [41, 42]. Despite the RO5263397-induced enhancement in wakefulness, RO5263397 neither improved nor impaired WM as assessed by choice accuracy of DMTS performance at either delay interval or when delay intervals were extended up to $100 \mathrm{~s}$ (see Supplementary Information). As expected, all macaques took longer to respond when the cognitive workload was increased (long vs. short delay). RO5263397 was well tolerated and the macaques were motivated to perform the task since no changes in number of omissions occurred. In a previous study, we demonstrated that RO5263397 (1-10 mg/kg, p.o.) improved accuracy on an object retrieval paradigm, a test of executive function mediated by the PFC [43, 44]. Collectively, these results suggest that TAAR 1 partial agonists may promote some aspects of executive function (e.g., response inhibition) more effectively than WM, at least as tested under the current task conditions. It is possible that increasing the task difficulty (e.g., increasing distractors) could reveal a bigger dynamic range in the paradigm [45], especially in the longer delay $(10 \mathrm{sec})$ condition and thus allow for greater modulation in performance following RO5263397 administration.

Together, our results indicate that, when administered at a time of day when sleep pressure is high, the TAAR1 partial agonist RO5263397 enhances wakefulness, suppresses NREM/REM sleep and alters EEG spectral composition in Cynomolgus macaques without inducing hyperlocomotion. These properties suggest the utility of TAAR1-directed compounds in treating sleep disorders such as narcolepsy, in which excessive sleepiness and REM sleep abnormalities are characteristic. 


\section{FUNDING AND DISCLOSURE}

Research supported by NIH R21NS085757 and R01NS103529. M.C.H. is an employee of F. Hoffmann-La Roche Ltd. T.L.W. is an employee of Blackthorn Therapeutics. Over the past 2 years, T.S.K. has served as a consultant for $\mathrm{NIH}$, the Japanese Society for the Promotion of Science, Teva Branded Pharmaceutical Products $R \& D$, Inc. and Jazz Pharmaceuticals and received research support from Jazz Pharmaceuticals and Sunovion Pharmaceuticals, Inc. S.R. $M$. has received research support from Neurocrine Biosciences, Unity Biotechnology, Verily Life Sciences LLC, Teva Branded Pharmaceutical Products R\&D, Inc., Jazz Pharmaceuticals, Heptares Therapeutics and Sunovion Pharmaceuticals, Inc. The remaining authors declare no conflict of interests.

\section{ACKNOWLEDGEMENTS}

We thank the SRI Laboratory Animal Medicine Department for excellent veterinary care and husbandry of the animals and Drs. Massimiliano de Zambotti and Michael Schwartz for comments on the manuscript. We also thank Dr. Joerg Hipp of F. Hoffmann-La Roche Ltd. for supplying the visual stimuli used in the DMTS study.

\section{ADDITIONAL INFORMATION}

Supplementary Information accompanies this paper at (https://doi.org/10.1038/ s41386-019-0386-8).

Publisher's note: Springer Nature remains neutral with regard to jurisdictional claims in published maps and institutional affiliations.

\section{REFERENCES}

1. Burchett SA, Hicks TP. The mysterious trace amines: protean neuromodulators of synaptic transmission in mammalian brain. Prog Neurobiol. 2006;79:223-46.

2. Grandy DK. Trace amine-associated receptor 1-Family archetype or iconoclast? Pharmacol Ther. 2007;116:355-90.

3. Gainetdinov RR, Hoener MC, Berry MD. Trace amines and their receptors. Pharmacol Rev. 2018;70:549-620.

4. Borowsky B, Adham N, Jones KA, Raddatz R, Artymyshyn R, Ogozalek KL, et al. Trace amines: identification of a family of mammalian $G$ protein-coupled receptors. Proc Natl Acad Sci USA. 2001;98:8966-71.

5. Bunzow JR, Sonders MS, Arttamangkul S, Harrison LM, Zhang G, Quigley DI, et al. Amphetamine, 3,4-methylenedioxymethamphetamine, lysergic acid diethylamide, and metabolites of the catecholamine neurotransmitters are agonists of a rat trace amine receptor. Mol Pharmacol. 2001;60:1181-8.

6. Berry MD, Gainetdinov RR, Hoener MC, Shahid M. Pharmacology of human trace amine-associated receptors: therapeutic opportunities and challenges. Pharmacol Ther. 2017;180:161-80.

7. Lindemann L, Meyer CA, Jeanneau K, Bradaia A, Ozmen L, Bluethmann H, et al. Trace amine-associated receptor 1 modulates dopaminergic activity. J Pharmacol Exp Ther. 2008;324:948-56.

8. Xie Z, Miller GM. Trace amine-associated receptor 1 is a modulator of the dopamine transporter. J Pharmacol Exp Ther. 2007;321:128-36.

9. Revel FG, Moreau JL, Gainetdinov RR, Ferragud A, Velazquez-Sanchez C, Sotnikova TD, et al. Trace amine-associated receptor 1 partial agonism reveals novel paradigm for neuropsychiatric therapeutics. Biol Psychiatry. 2012;72:934-42.

10. Revel FG, Moreau JL, Pouzet B, Mory R, Bradaia A, Buchy D, et al. A new perspective for schizophrenia: TAAR1 agonists reveal antipsychotic- and antidepressant-like activity, improve cognition and control body weight. Mol Psychiatry. 2013;18:543-56.

11. Schwartz MD, Black SW, Fisher SP, Palmerston JB, Morairty SR, Hoener MC, et al. Trace amine-associated receptor 1 regulates wakefulness and EEG spectral composition. Neuropsychopharmacology. 2017;42:1305-14.

12. Black SW, Schwartz MD, Chen T-M, Hoener MC, Kilduff TS. TAAR1 agonists as narcolepsy therapeutics. Biol Psychiatry. 2017;82:623-33.

13. Edgar DM, Dement WC, Fuller CA. Effect of SCN lesions on sleep in squirrel monkeys: evidence for opponent processes in sleep-wake regulation. J Neurosci. 1993;13:1065-79.

14. Klerman EB, Boulos Z, Edgar DM, Mistlberger RE, Moore-Ede MC. Circadian and homeostatic influences on sleep in the squirrel monkey: sleep after sleep deprivation. Sleep. 1999;22:45-59.

15. Daley JT, Turner RS, Freeman A, Bliwise DL, Rye DB. Prolonged assessment of sleep and daytime sleepiness in unrestrained Macaca mulatta. Sleep. 2006;29:221-31
16. Darbin O, Smart O, Wichmann T. A non-invasive technique to monitor wakefulness during electrophysiologic recording experiments in primates. J Neurosci Methods. 2009;177:448-51.

17. Hsieh KC, Robinson EL, Fuller CA. Sleep architecture in unrestrained rhesus monkeys (Macaca mulatta) synchronized to 24-hour light-dark cycles. Sleep. 2008:31:1239-50.

18. Tannenbaum PL, Tye SJ, Stevens J, Gotter AL, Fox SV, Savitz AT, et al. Inhibition of orexin signaling promotes sleep yet preserves salient arousability in monkeys. Sleep. 2016;39:603-12.

19. Authier S, Paquette D, Gauvin D, Sammut V, Fournier S, Chaurand F, et al. Videoelectroencephalography in conscious non human primate using radiotelemetry and computerized analysis: refinement of a safety pharmacology model. J Pharmacol Toxicol Methods. 2009;60:88-93.

20. Rachalski A, Authier S, Bassett L, Pouliot M, Tremblay G, Mongrain V. Sleep electroencephalographic characteristics of the Cynomolgus monkey measured by telemetry. J Sleep Res. 2014;23:619-27.

21. Goonawardena A, Morairty SR, Orellana GA, Willoughby AR, Wallace TL, Kilduff TS. Electrophysiological characterization of sleep/wake, activity and the response to caffeine in adult cynomolgus macaques. Neurobiol Sleep Circadian Rhythms. 2019;6:9-23.

22. Balzamo E, Santucci V, Seri B, Vuillon-Cacciuttolo G, Bert J. Nonhuman primates: laboratory animals of choice for neurophysiologic studies of sleep. Lab Anim Sci. 1977;27(5 Pt 2):879-86.

23. Uslaner JM, Tye SJ, Eddins DM, Wang X, Fox SV, Savitz AT, et al. Orexin receptor antagonists differ from standard sleep drugs by promoting sleep at doses that do not disrupt cognition. Sci Transl Med. 2013;5:179ra44.

24. Miller GM, Verrico CD, Jassen A, Konar M, Yang $H$, Panas $H$, et al. Primate trace amine receptor 1 modulation by the dopamine transporter. J Pharmacol Exp Ther. 2005;313:983-94

25. Iber C, Ancoli-Israel S, Chesson A, Quan SF. The AASM manual for the scoring of sleep and associated events: rules, terminology and technical specifications. 1st ed. Westchester: American Academy of Sleep Medicine; 2007.

26. Silber MH, Ancoli-Israel S, Bonnet MH, Chokroverty S, Grigg-Damberger MM, Hirshkowitz $M$, et al. The visual scoring of sleep in adults. J Clin Sleep Med. 2007:3:121-31.

27. Goonawardena AV, Heiss J, Glavis-Bloom C, Trube G, Borroni E, Alberati D, et al. Alterations in high-frequency neuronal oscillations in a Cynomolgus macaque test of sustained attention following NMDA receptor antagonism. Neuropsychopharmacology. 2016;41:1319-28.

28. Espinoza S, Salahpour A, Masri B, Sotnikova TD, Messa M, Barak LS, et al. Functional interaction between trace amine-associated receptor 1 and dopamine D2 receptor. Mol Pharmacol. 2011;80:416-25.

29. Leo D, Mus L, Espinoza S, Hoener MC, Sotnikova TD, Gainetdinov RR. Taar1mediated modulation of presynaptic dopaminergic neurotransmission: role of D2 dopamine autoreceptors. Neuropharmacology. 2014;81:283-91.

30. Revel FG, Moreau JL, Gainetdinov RR, Bradaia A, Sotnikova TD, Mory R, et al. TAAR1 activation modulates monoaminergic neurotransmission, preventing hyperdopaminergic and hypoglutamatergic activity. Proc Natl Acad Sci USA. 2011;108:8485-90.

31. Bradaia A, Trube G, Stalder H, Norcross RD, Ozmen L, Wettstein JG, et al. The selective antagonist EPPTB reveals TAAR1-mediated regulatory mechanisms in dopaminergic neurons of the mesolimbic system. Proc Natl Acad Sci USA. 2009;106:20081-6.

32. Mignot E, Nishino S, Guilleminault C, Dement WC. Modafinil binds to the dopamine uptake carrier site with low affinity. Sleep. 1994;17:436-7.

33. Hasan S, Pradervand S, Ahnaou A, Drinkenburg W, Tafti M, Franken P. How to keep the brain awake? The complex molecular pharmacogenetics of wake promotion. Neuropsychopharmacology. 2009;34:1625-40.

34. Schwartz MD, Palmerston JB, Lee DL, Hoener MC, Kilduff TS. Deletion of trace amine-associated receptor 1 attenuates behavioral responses to caffeine. Front Pharmacol. 2018;9:35.

35. Touret M, Sallanon-Moulin M, Fages C, Roudier V, Didier-Bazes M, Roussel B, et al. Effects of modafinil-induced wakefulness on glutamine synthetase regulation in the rat brain. Brain Res Mol Brain Res. 1994;26:123-8.

36. Espinoza S, Lignani G, Caffino L, Maggi S, Sukhanov I, Leo D, et al. TAAR1 modulates cortical glutamate NMDA receptor function. Neuropsychopharmacology. 2015;40:2217-27.

37. Ehrlichman RS, Gandal MJ, Maxwell CR, Lazarewicz MT, Finkel LH, Contreras D, et al. N-methyl-d-aspartic acid receptor antagonist-induced frequency oscillations in mice recreate pattern of electrophysiological deficits in schizophrenia. Neuroscience. 2009;158:705-12.

38. Hakami T, Jones NC, Tolmacheva EA, Gaudias J, Chaumont J, Salzberg M, et al. NMDA receptor hypofunction leads to generalized and persistent aberrant gamma oscillations independent of hyperlocomotion and the state of consciousness. PloS one. 2009;4:e6755. 
Trace amine-associated receptor 1 agonism promotes wakefulness without... AV Goonawardena et al.

39. Lazarewicz MT, Ehrlichman RS, Maxwell CR, Gandal MJ, Finkel LH, Siegel SJ. Ketamine modulates theta and gamma oscillations. J Cogn Neurosci. 2010;22:1452-64.

40. Pinault D. N-methyl d-aspartate receptor antagonists ketamine and MK-801 induce wake-related aberrant gamma oscillations in the rat neocortex. Biol Psychiatry. 2008;63:730-5.

41. Li BM, Mei ZT. Delayed-response deficit induced by local injection of the alpha 2 -adrenergic antagonist yohimbine into the dorsolateral prefrontal cortex in young adult monkeys. Behav Neural Biol. 1994;62:134-9.

42. Sawaguchi T, Goldman-Rakic PS. D1 dopamine receptors in prefrontal cortex: involvement in working memory. Science. 1991;251:947-50.
43. Jentsch JD, Taylor JR, Elsworth JD, Redmond DE Jr, Roth RH. Altered frontal cortical dopaminergic transmission in monkeys after subchronic phencyclidine exposure: involvement in frontostriatal cognitive deficits. Neuroscience. 1999; 90:823-32.

44. Rutten K, Basile JL, Prickaerts J, Blokland A, Vivian JA. Selective PDE inhibitors rolipram and sildenafil improve object retrieval performance in adult cynomolgus macaques. Psychopharmacology. 2008;196:643-8.

45. Porrino LJ, Daunais JB, Rogers GA, Hampson RE, Deadwyler SA. Facilitation of task performance and removal of the effects of sleep deprivation by an ampakine (CX717) in nonhuman primates. PLoS Biol. 2005;3:e299. 